Supporting Information

\title{
Rotational Periodicity Display of the Tunable Wettability Pattern in a Photoswitch Based on a Response Bilayer Photonic Crystal
}

Yong Qi, Wenbin Niu, Shufen Zhang*, Zhongjian Zhang, Suli Wu, and Wei Ma

State Key Laboratory of Fine Chemicals, Dalian University of Technology, P.O. Box 89, West Campus, 2\# Linggong Rd, Dalian 116024, China

*E-mail: zhangshf@dlut.edu.cn

Schematic diagram of the forward and backward diffraction spectra detection of the photonic crystals. Figure 1g, S4, and S9 were detected using model Figure S1a. The incident angle in Figure $1 \mathrm{~g}$ and S4 were $5^{\circ}$. Figure $1 \mathrm{~h}, 3 \mathrm{~b}, 3 \mathrm{c}, 4 \mathrm{a}, 4 \mathrm{~b}, \mathrm{~S} 5, \mathrm{~S} 6 \mathrm{~b}, \mathrm{~S} 8$, and S10 were all detected using model Figure S1b. The incident angle is $57.5^{\circ}$, and the angle between detector and incident light is $10^{\circ}$. Figure S12a was detected using the model Figure S1d. Figure S12b was detected using the model Figure S1c. Figure 2a, 2e, 4c, 4d, 7b, 7c, 7d, 7e, and S18 were detected using model Figure S2a. The incident angle in Figure $7 \mathrm{~b}$ and $7 \mathrm{c}$ were $0^{\circ}$. The incident angle in Figure $7 \mathrm{~d}$ and $7 \mathrm{e}$ were $\sim 60^{\circ}$. Figure $2 \mathrm{c}$ and $2 \mathrm{~d}$ was detected using model Figure $\mathrm{S} 2 \mathrm{~b}$. 


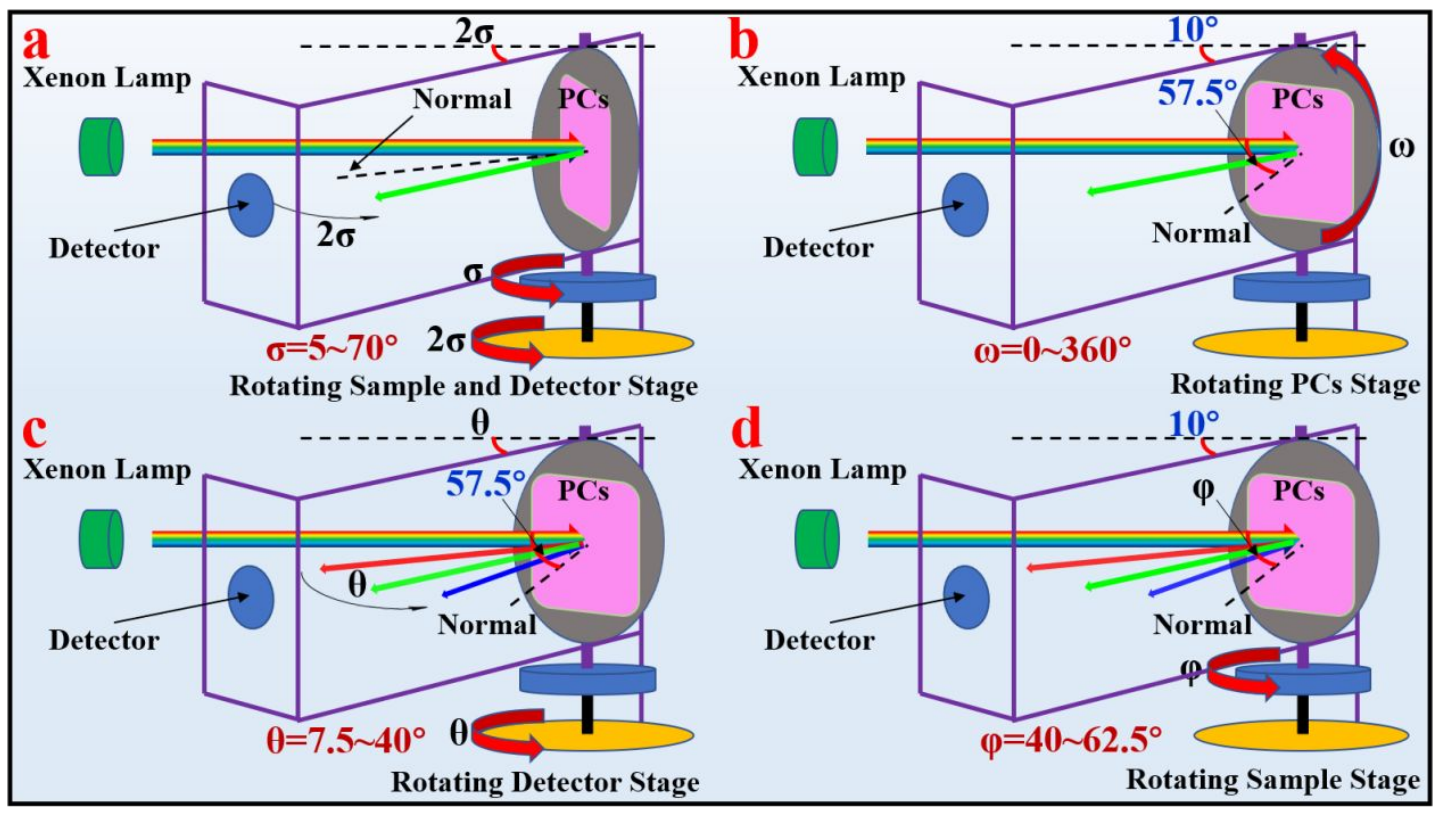

Figure S1. The schematic diagram of the optical spectrum detection of the rotational photoswitches, (a) detecting the 3D photonic bandgap (forward diffraction), (b) detecting the periodic 2D photonic bandgap (backward diffraction); The schematic diagram of the 2D scattering detection, (c) changing detection angle, (d) changing incidence angle.

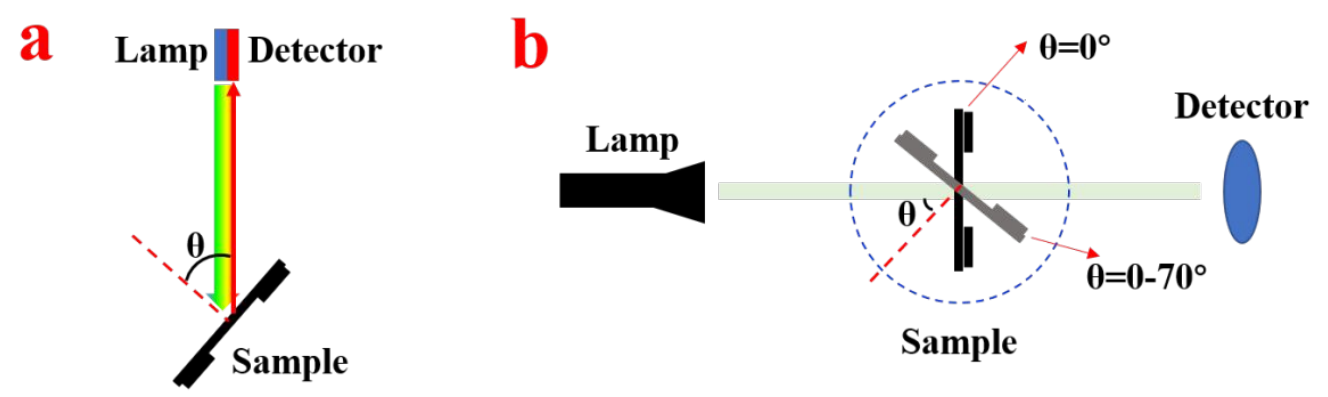

Figure S2. (a) The schematic diagram of the 2D optical (backward diffraction) angle dependence of the bilayer photonic crystals by using a spectrophotometer (Hitachi U-4100, Japan) with a Fiber Optic Spectrometer (EQ 2000). The light source is in the same position as the detector. The photo is taken in a similar way (change the light source to a flashlight and the detector to a camera). (b) The schematic diagram of the transmittance spectra of the films. 


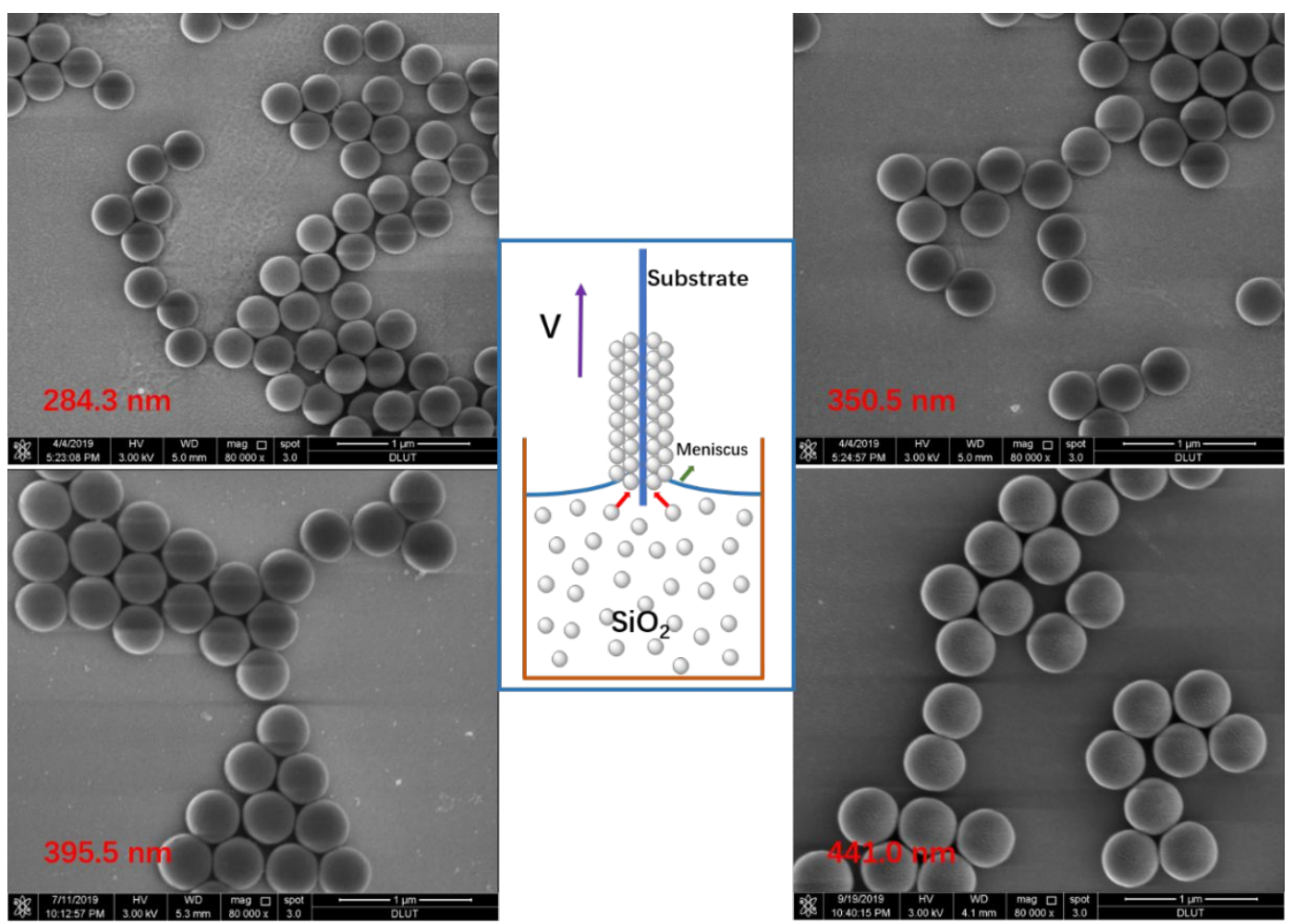

Figure S3. The $\mathrm{SiO}_{2}$ monodisperse nanospheres and the schematic illustration of the dipcoating method. $\sim 8 \mathrm{wt} \% \mathrm{SiO}_{2}$ ethanol dispersion was prepared for further use. The pulling rate of the dip-coating assembly process was $2 \mu \mathrm{m} \mathrm{s}^{-1}$ at $20^{\circ} \mathrm{C}$.

According to Bragg's law and Snell's law, the photonic stop bands of FCC structured PCs can be theoretically calculated as:

$$
\begin{gathered}
\lambda_{\max }=1.63 D\left(n_{e f f}{ }^{2}-\sin ^{2} \theta\right)^{1 / 2} \\
n_{e f f}{ }^{2}=0.74 \times n_{S i O 2^{2}}+0.26 \times n_{\text {air }}{ }^{2}
\end{gathered}
$$

Where $\lambda_{\max }$ is the maximum reflected wavelength, $\theta$ is the incident angle, $D$ is the diameter of the $\mathrm{SiO}_{2}$ nanospheres; $n_{\text {eff }}$ is the effective refractive index; $n_{\mathrm{SiO} 2}=1.45$ and $n_{\text {air }}=1.0$ are the refractive index of silica and air, respectively. We can prepare some value of silica nanospheres we need through the Bragg's law of diffraction. The formula can be converted to:

$$
D=\lambda_{\max } /\left[1.63 \times\left(1.82-\sin ^{2} \theta\right)^{1 / 2}\right]
$$

According to the maximum reflection wavelength in Figure S4, the particle size of silica nanoparticles can be roughly estimated, and the estimated value is close to the actual value determined by SEM (see Table S1 and Figure S4). 
Table S1. The diameter of the $\mathrm{SiO}_{2}$ particles (theoretical and practical)

\begin{tabular}{cccc}
\hline$\lambda_{\max }(\mathrm{nm})$ & $\theta\left(^{\circ}\right)$ & Theoretical $D(\mathrm{~nm})$ & Practical $D(\mathrm{~nm})$ \\
\hline 652.0 & 5 & 296.6 & $\sim 284.3$ \\
778.5 & 5 & 354.2 & $\sim 350.5$ \\
872.0 & 5 & 397.4 & $\sim 395.5$ \\
977.0 & 5 & 445.2 & $\sim 441.0$ \\
\hline
\end{tabular}

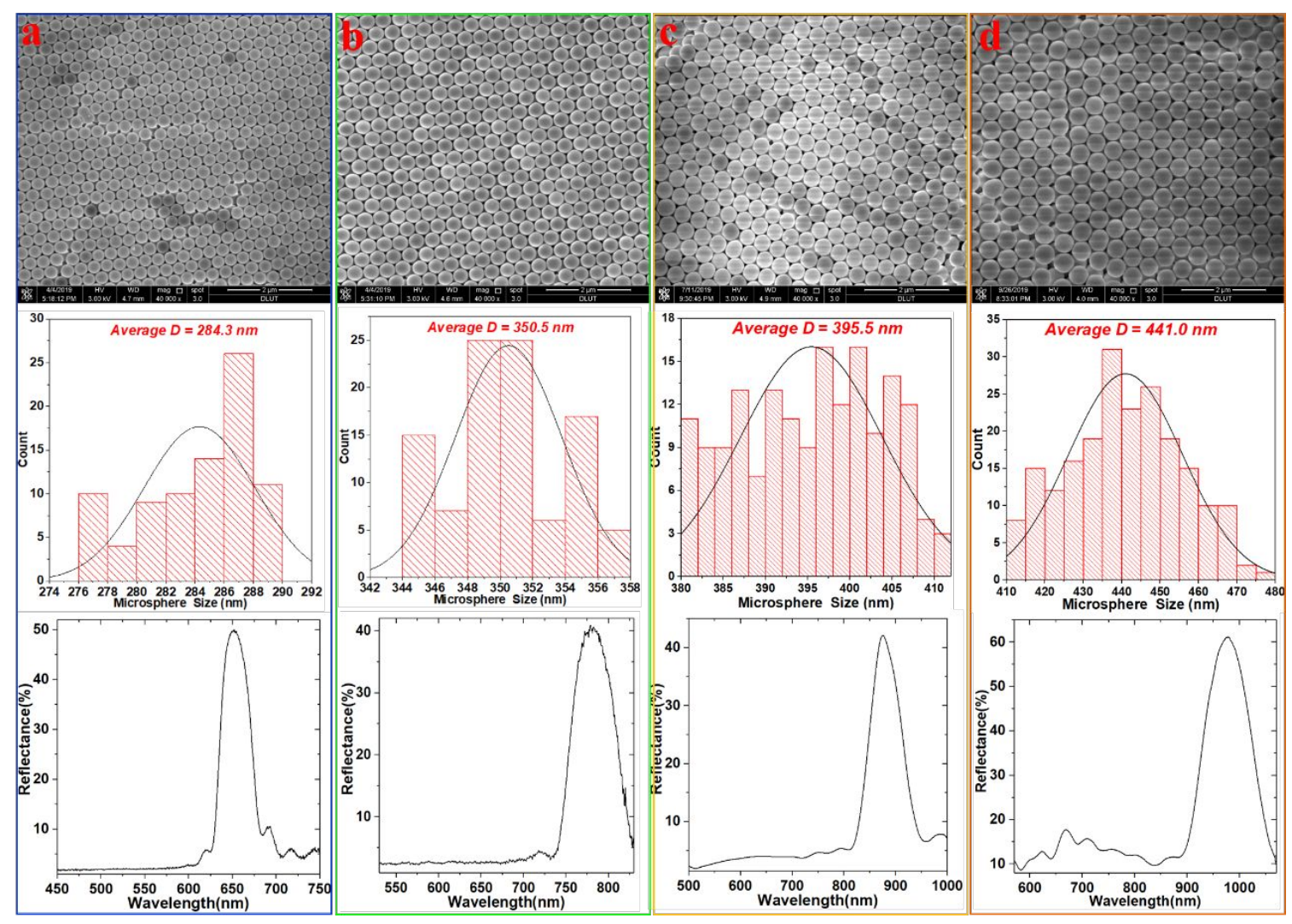

Figure S4. The SEM (top view) of opal photonic crystals, the corresponding particle size statistic, and the corresponding 3D reflection spectrum. (a) $284.3 \mathrm{~nm}$, (b) $\sim 350.5 \mathrm{~nm}$, (c) $\sim 395.5 \mathrm{~nm},(\mathrm{~d}) \sim 441.0 \mathrm{~nm}$. 


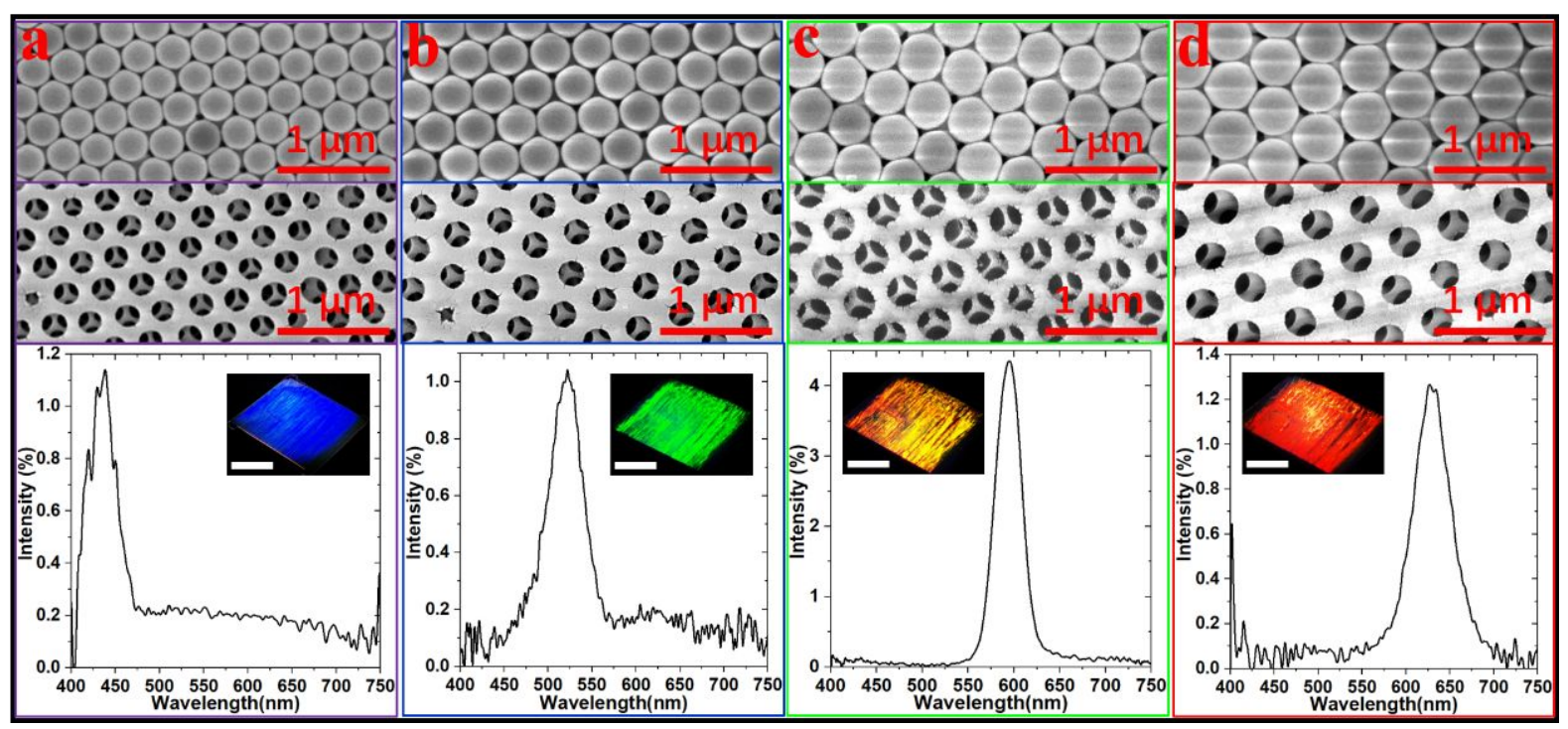

Figure S5. Typical SEM images (top view) of the opals and the inverse opals with different particle sizes and the corresponding 2D optical bandgap of the inverse opals. (b) $\sim 285.3 \mathrm{~nm}$, (c) $\sim 350.5 \mathrm{~nm}$, (d) $\sim 395.5 \mathrm{~nm}$, (e) $\sim 441.0 \mathrm{~nm}$. The insets are the corresponding digital photos (the scale bars are $1 \mathrm{~cm}$ ).
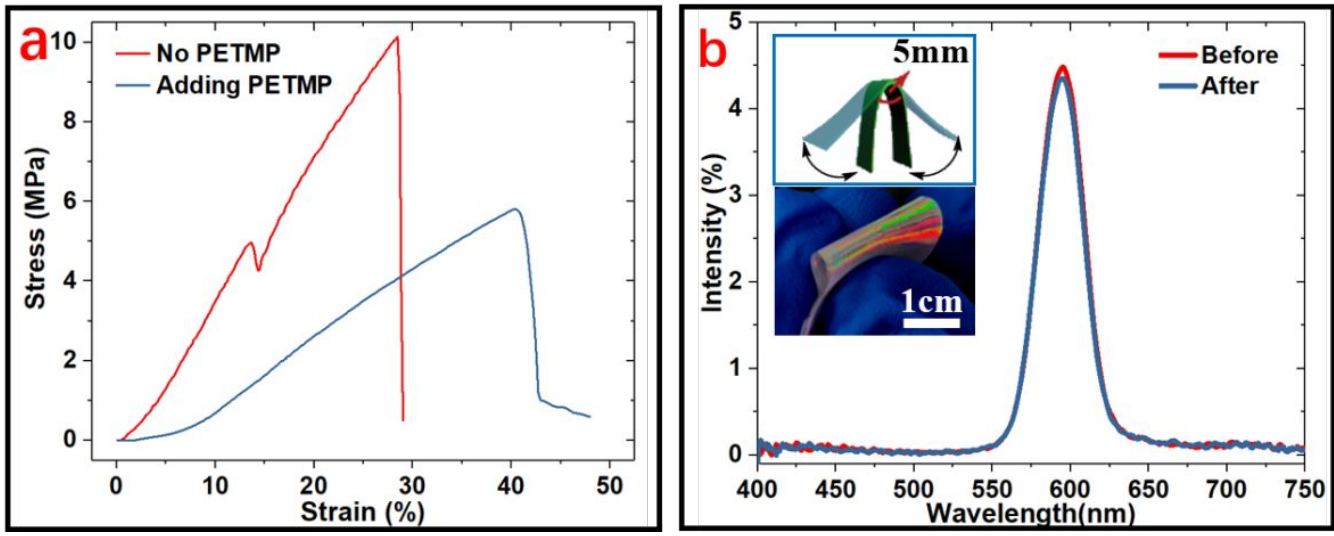

Figure S6. Tensile strain curves and bending test diagrams of the photoswitch. (a) Effects of PETMP on the tensile strain of the bilayer film. It was found that adding an appropriate amount of PETMP was beneficial to improve the flexibility of the film. (b) The 2D reflectance spectrum changes at the transversely folded part before and after the film bending test. The bilayer film (with a thickness of approximately $0.3 \mathrm{~mm}$ ) was clamped tightly by the test fixtures (inset), and the fixtures stretched the film gradually at a rate of $5 \mathrm{~mm} / \mathrm{min}$. The reflection spectrum barely changed after 300 bending cycles ("A-B Mode"). 


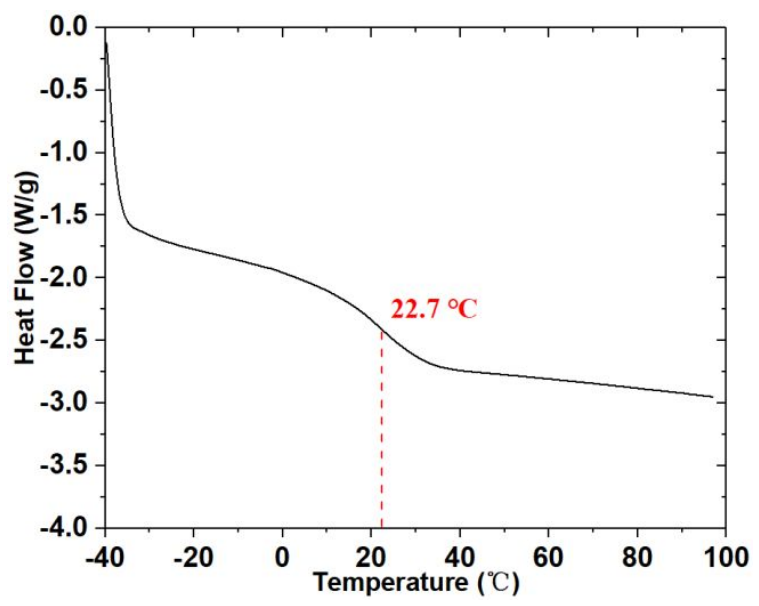

Figure S7. Differential scanning calorimetry plot of an ETPTA-co-PEGDA-co-AA-coPETMP copolymer with 1:4:1:0.25 ratio. A single $T_{g}$ of $\sim 22.7^{\circ} \mathrm{C}$ measured by differential scanning calorimetry of a bilayer photonic crystal indicates the cross-linked copolymer is a homogeneous mixture of the three components.

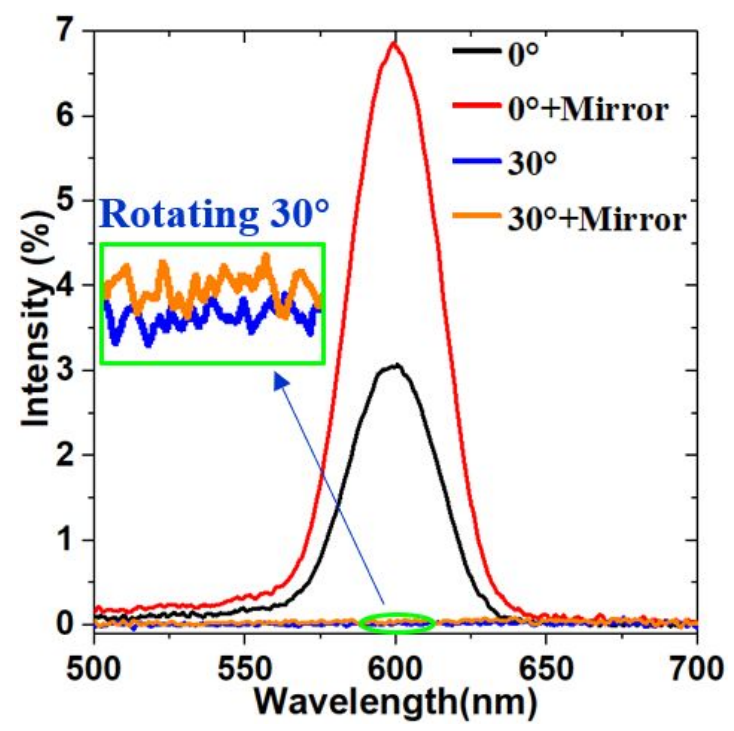

Figure S8. The backward diffraction of the inverse opal (with or without a mirror). After rotation of $30^{\circ}$, all of the PBG disappear. By placing a mirror under the photonic crystal, the backward diffraction intensity of the 3D inverse opal can be greatly improved. It indicates that the direction of this backward diffraction includes up and down. 


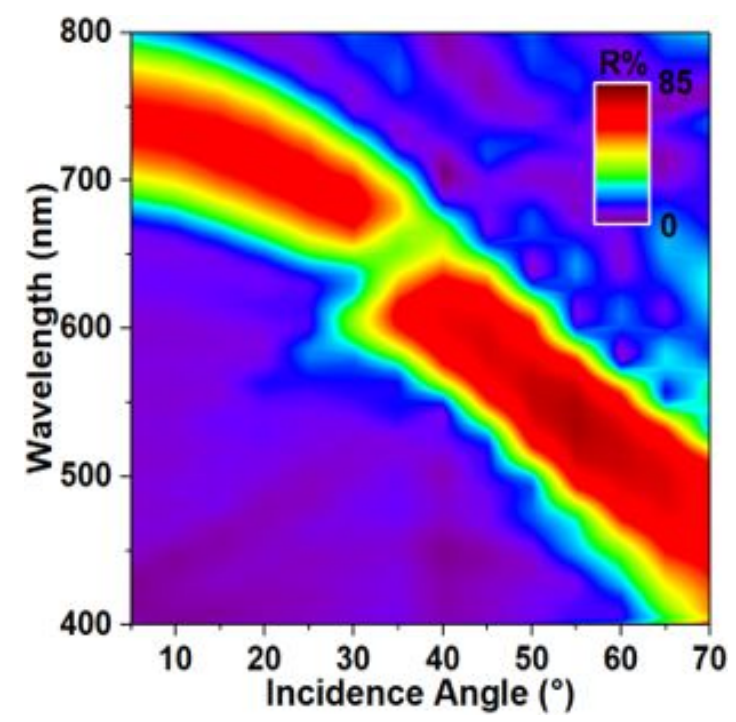

Figure S9. The relation between the maximum reflected wavelength of the forward diffraction and the incident angle.
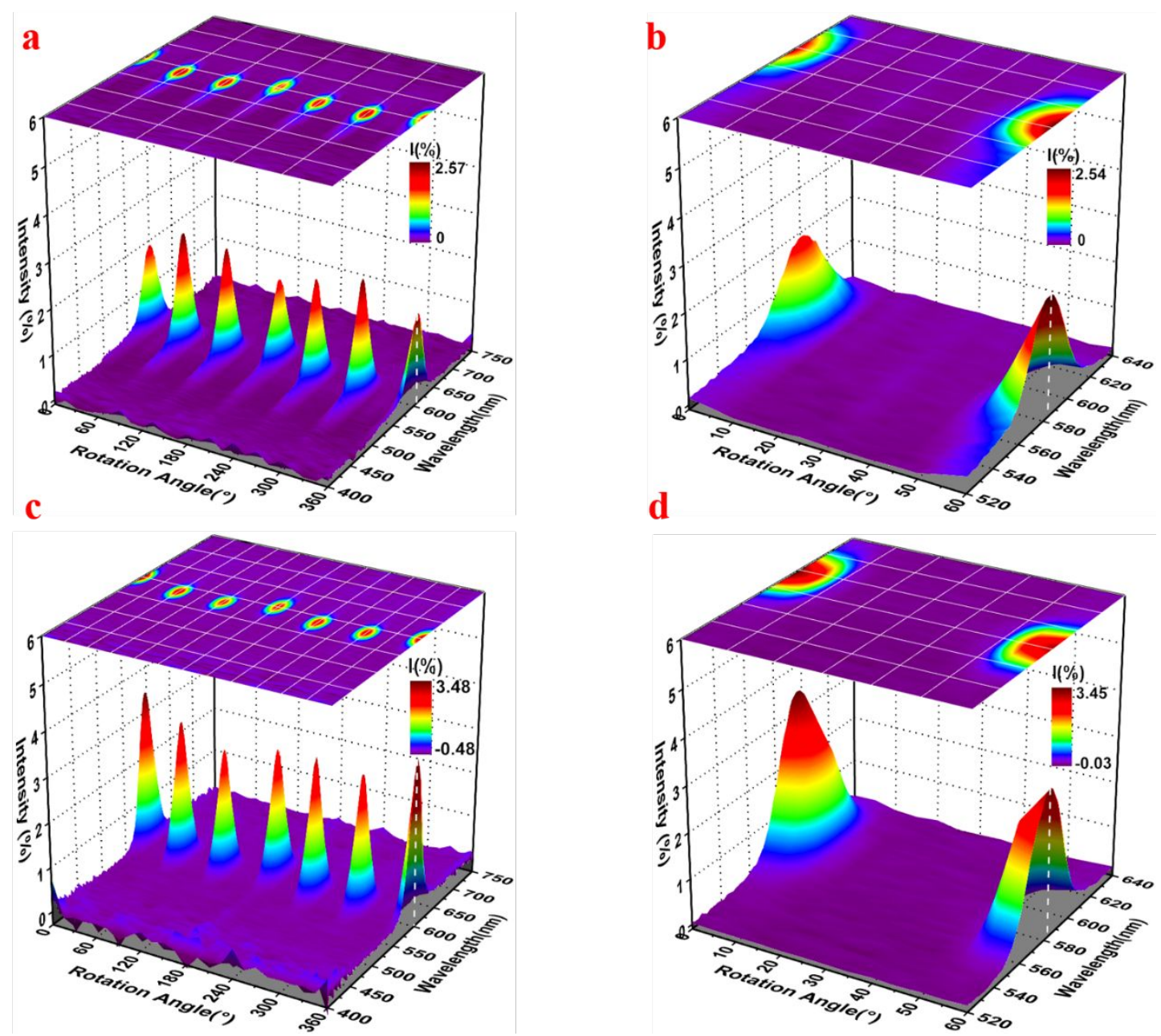

Figure S10. The periodic change of 2D bandgap (backward diffraction) during rotation of the opal or inverse opal photonic crystal. The detection method is shown in Figure 2c. (a) the 
changing bandgap of the opal during the $360^{\circ}$ rotation; (b) the changing bandgap of the opal during the $60^{\circ}$ rotation; (c) the changing bandgap of the inverse opal during the $360^{\circ}$ rotation; (d) the changing bandgap of the inverse opal during the $60^{\circ}$ rotation. It was found that the structure color of 2D diffraction displays or disappears periodically when the opal or inverse opal photonic crystals rotate along the film plane. The period of the photonic bandgap is $60^{\circ}$.
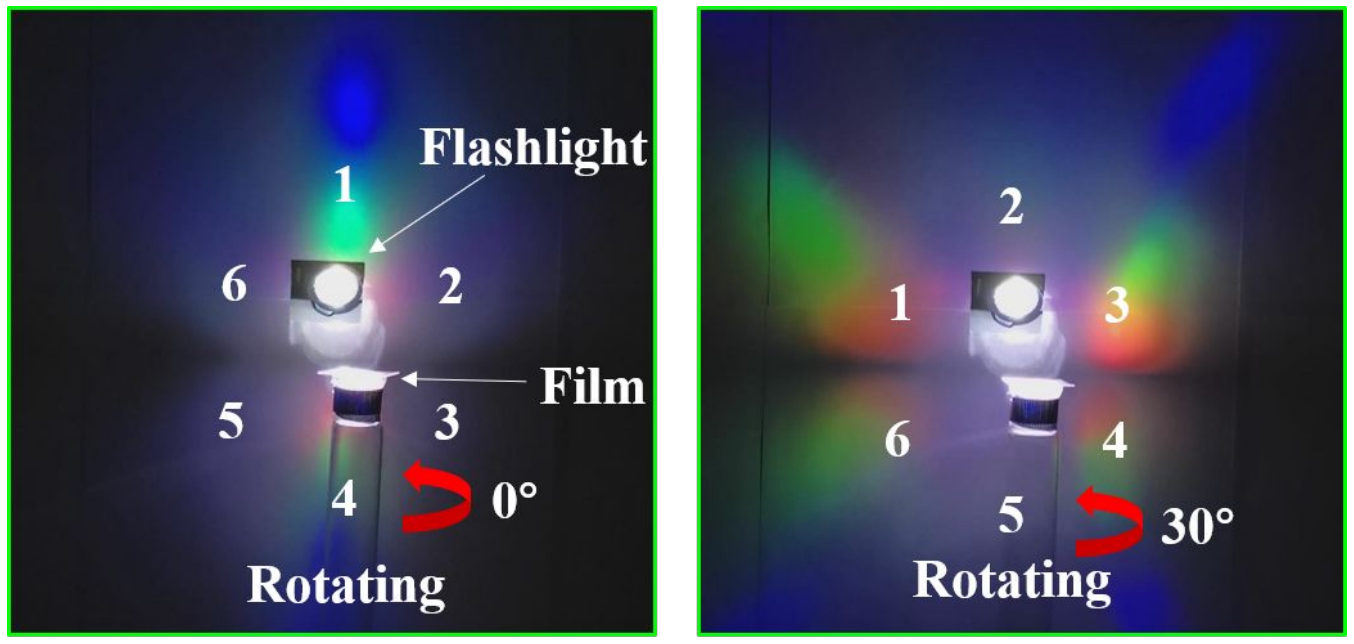

Figure S11. The 2D diffraction imaging of the monolayer inverse opal at different rotation angles, the incidence angle was $\sim 60^{\circ}$.
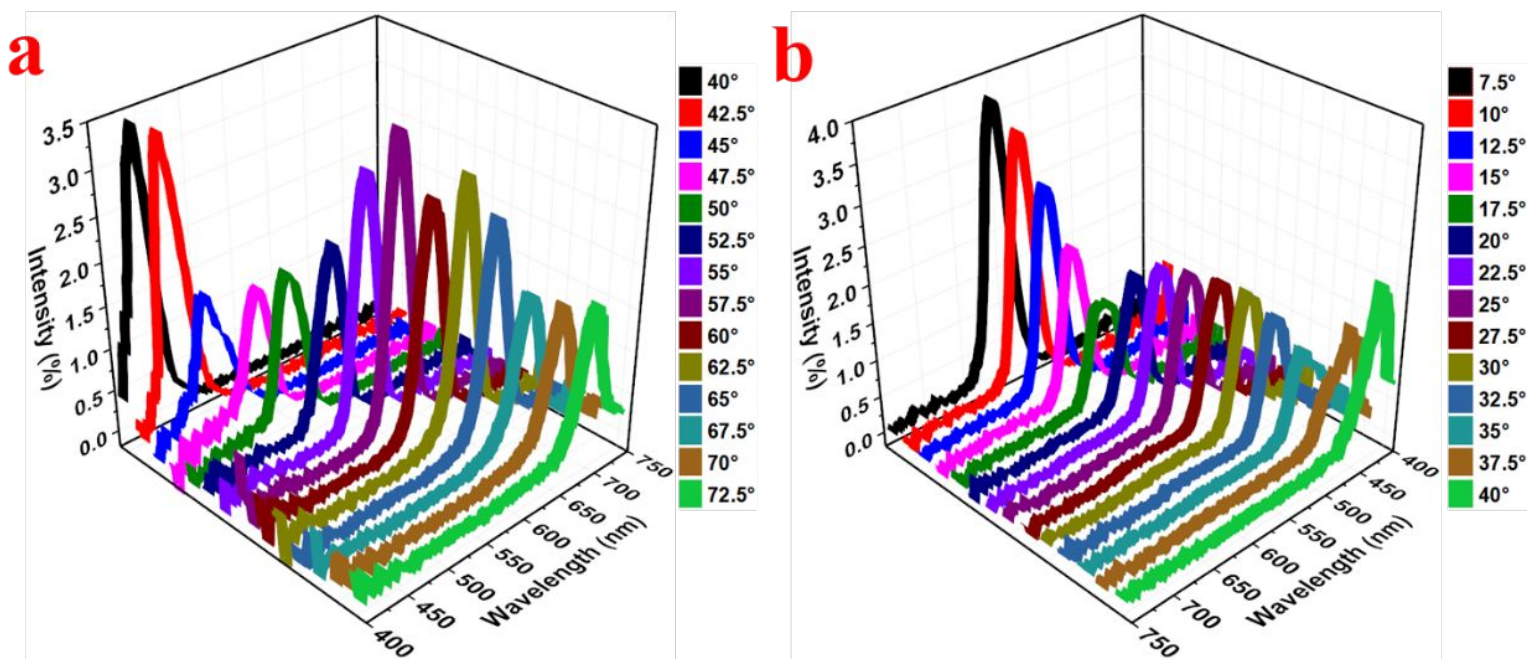

Figure S12. (a) The relationship between the 2D scattering and the incidence angle. The included angle between the incident light and the detector is $10^{\circ}$. As the incidence angle 
increases, the photonic bandgap shows redshift from $\sim 430 \mathrm{~nm}$ to $\sim 690 \mathrm{~nm}$. (b) The relationship between the $2 \mathrm{D}$ scattering and the detection angle. The incidence angle is $57.5^{\circ}$. The photonic bandgap shows blueshift from $\sim 610 \mathrm{~nm}$ to $\sim 420 \mathrm{~nm}$ with the increase of detection angle.

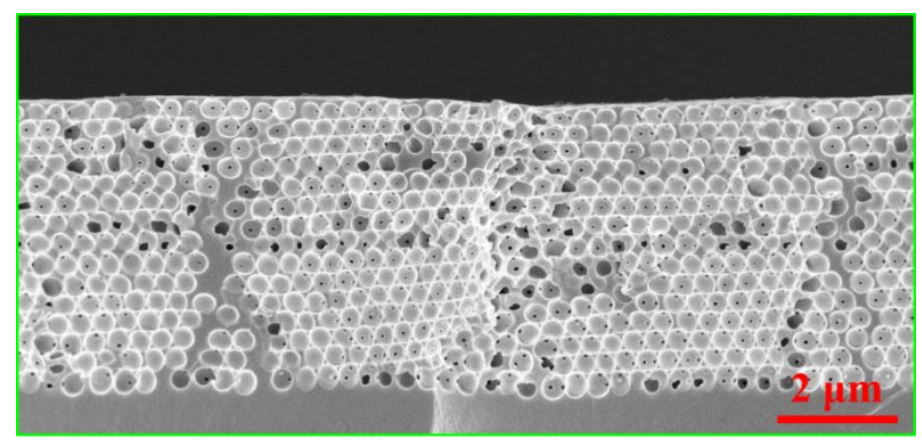

Figure S13. Typical cross-sectional SEM image of the inverse opal with a thickness of $\sim 4.87$ $\mu \mathrm{m}$.
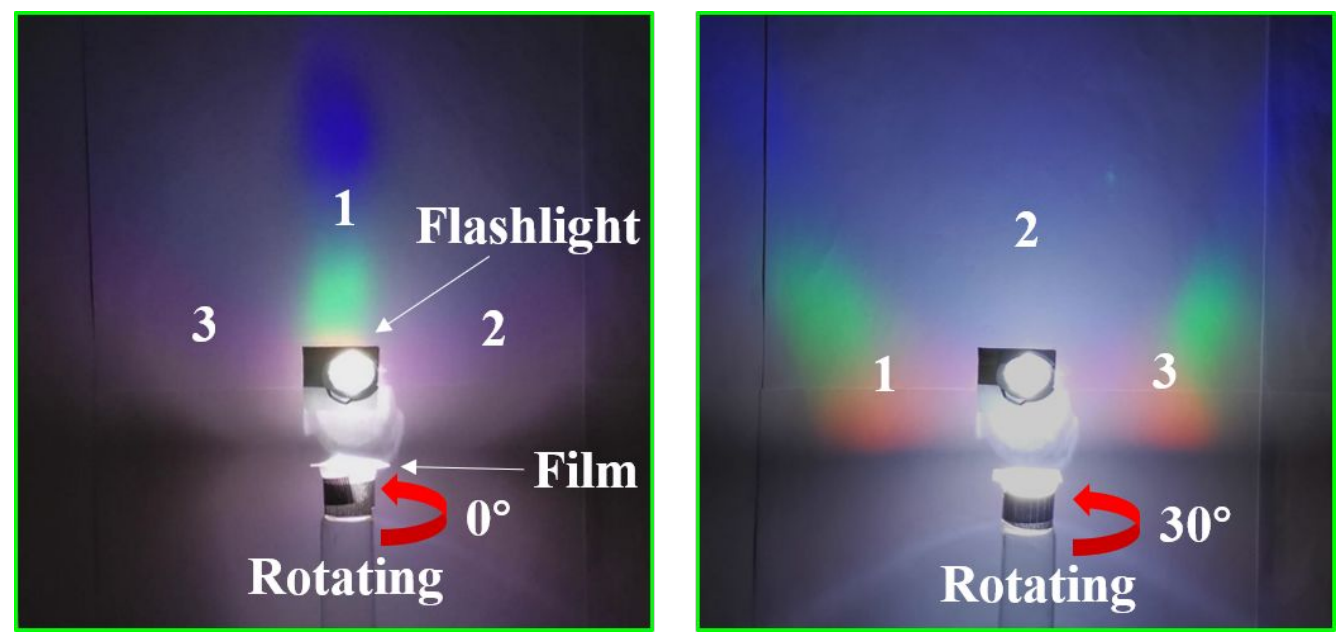

Figure S14. The 2D diffraction imaging of the bilayer photonic crystal (both of the top layer and the bottom layer are thick, $\sim 4.87 \mu \mathrm{m}$, Figure S14, Supporting Information) at different rotation angles, the incidence angle was $\sim 60^{\circ}$. 

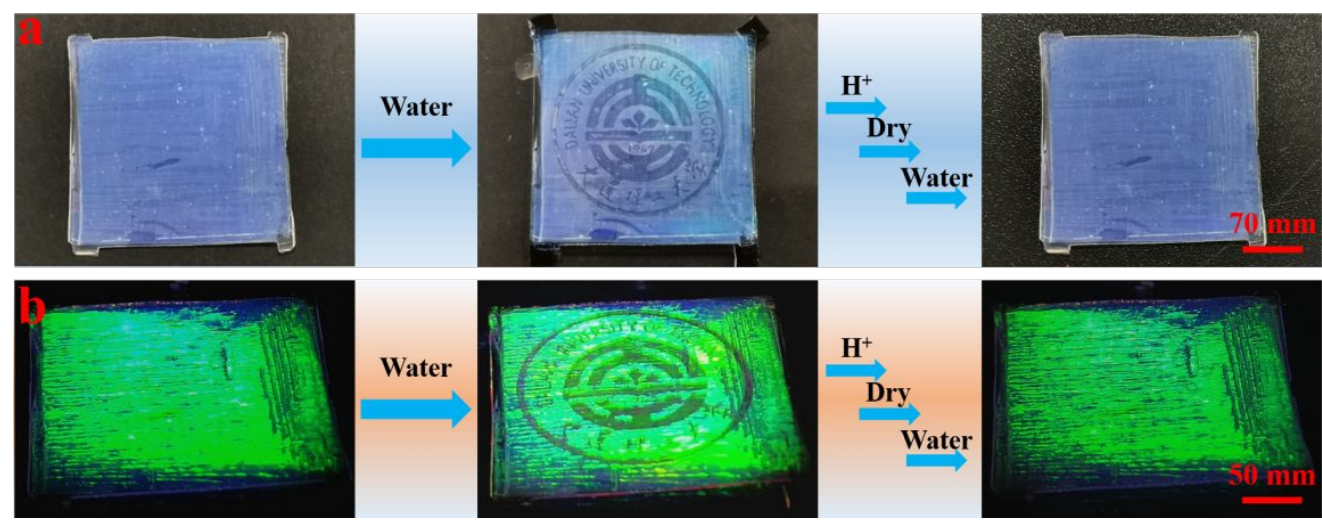

Figure S15. The revealing and resetting process of the wettability pattern during the water response. (a) Top view, no flashlight; (b) Flashlight incidence angle $\sim 50^{\circ}$, light source and camera in the same position. The pattern is obtained by stamping on the "Layer A" of the photoswitch after applying the seal with sodium carbonate solution $(\mathrm{pH} \approx 12)$. Resetting process of the wettability pattern: having been soaked in a solution of dilute hydrochloric acid $(\mathrm{pH} \approx 5)$ for $3 \mathrm{~min}$, the film was washed with distilled water three times. Finally, compressed air was used to dry (The university logo credit: Dalian University of Technology).
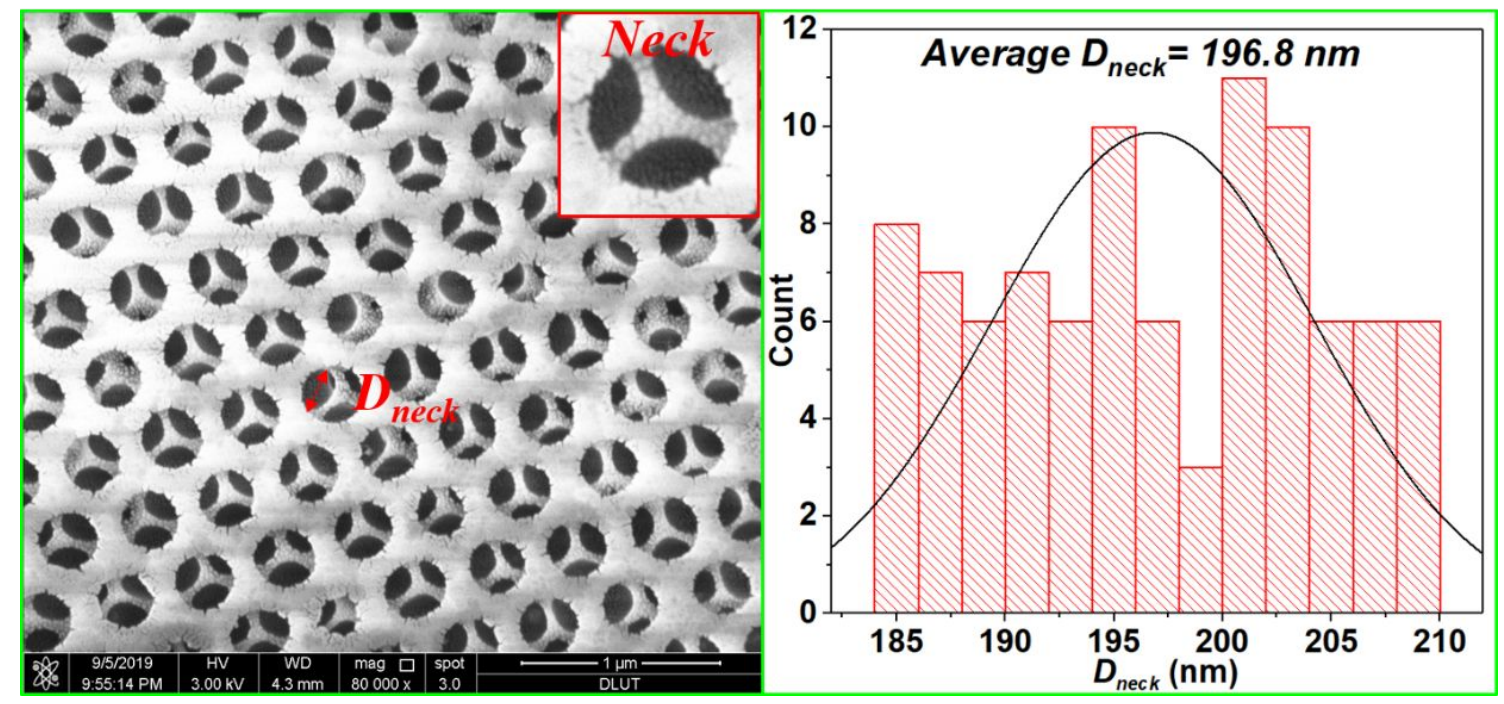

Figure S16. The neck diameters $\left(D_{\text {neck }}\right)$ are subject to the top view of the SEM. The statistics of the neck diameter obtained from those maximum diameters of the great circle arc. The average $D_{\text {neck }}$ is $\sim 196.8 \mathrm{~nm}$. 

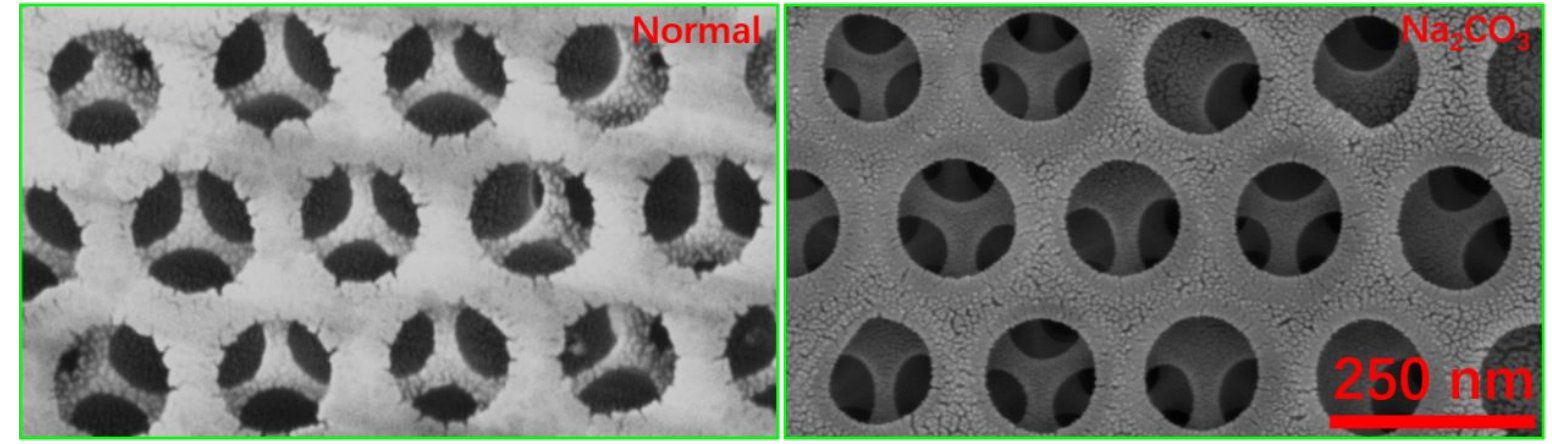

Figure S17. The typical SEM images of the bilayer photonic crystals before and after treatment with sodium carbonate solution. The surface morphology of the inverse opal was almost unchanged. For structural colors, the reflection spectra under the same conditions are nearly identical as long as the structure remains unchanged. It can be seen from Figure $7 \mathrm{~b}-\mathrm{e}$ (at $\mathrm{t}=0 \mathrm{~s}$ ) that before and after sodium carbonate treatment, under the same test conditions, the 2D backward diffraction and 3D forward diffraction spectra of the photoswitch are almost unchanged.

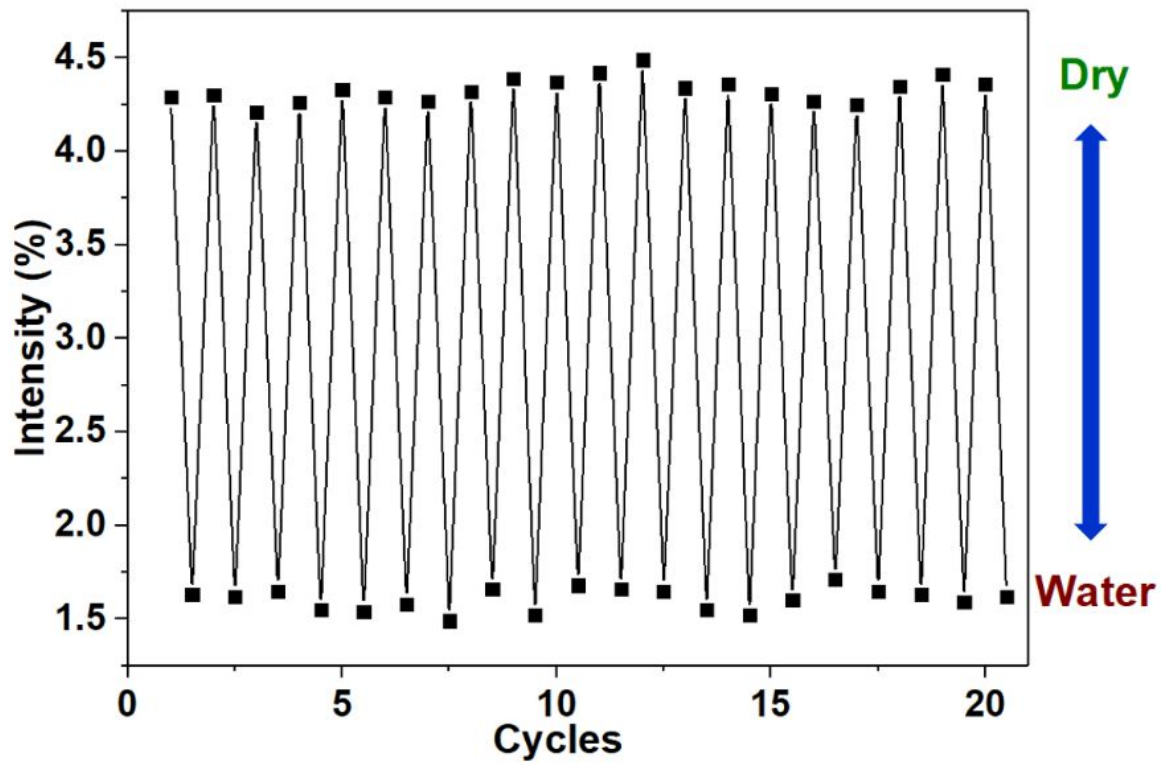

Figure S18. The cycles of the wettability pattern ("A-B Mode") in water-response and compressed air-drying. It has no obvious attenuation after repeated cycles. 
Supplementary Video S1. The 2D optical diffraction imaging of the inverse opal film. The incident angle is $\sim 60^{\circ}$.

Supplementary Video S2. The 2D optical diffraction imaging of the bilayer film ("A-B Mode"). The incident angle is $\sim 60^{\circ}$.

Supplementary Video S3. The 2D optical diffraction imaging of the bilayer film ("B-A Mode"). The incident angle is $\sim 60^{\circ}$.

Supplementary Video S4. The periodic bandgap of the bilayer film ("B-A Mode"). The light source is in the same position as the lens and the incident angle is $\sim 50^{\circ}$. When the inverse opal rotates $360^{\circ}$ along the plane of the film, the green "DUT" can be observed at the position of the light source for twelve times. The reproducibility period of the photonic bandgap is $30^{\circ}$ (The abbreviation “DUT" credit: Dalian University of Technology).

Supplementary Video S5. The periodic bandgap of the bilayer film ("A-B Mode"). The light source is in the same position as the lens and the incident angle is $\sim 50^{\circ}$. When the inverse opal rotates $360^{\circ}$ along the plane of the film, the green "DUT" can be observed at the position of the light source for six times. The reproducibility period of the photonic bandgap is $60^{\circ}$ (The abbreviation "DUT" credit: Dalian University of Technology).

Supplementary Video S6. The revelation of the invisible wettability pattern ("A-B Mode"). It is completely hidden under normal conditions, which will be revealed rapidly because of the decreasing of the refractive index differentials when immersed in water (The university logo credit: Dalian University of Technology). 
Supplementary Video S7. Rotational periodicity turning on and off of the revealed wettability pattern ("B-A Mode"). The periodical opening and closing of the superimposed bandgaps enable the revealed wettability pattern to be periodically displayed and hidden (The university logo credit: Dalian University of Technology). 